\title{
Constitutive modeling and experimental calibration of pressure effect for polyurea based on free volume concept
}

\author{
W. Nantasetphong ${ }^{\mathrm{a}, *}$, A. V. Amirkhizi ${ }^{\mathrm{b}}$, S. Nemat-Nasser ${ }^{\mathrm{a}}$ \\ ${ }^{a}$ Center of Excellence for Advanced Materials, Department of Mechanical and Aerospace \\ Engineering, University of California, San Diego, 9500 Gilman Drive, La Jolla, CA \\ 92093-0416 \\ ${ }^{b}$ Department of Mechanical Engineering, University of Massachusetts, Lowell, 1 \\ University Ave, Lowell, MA 01854
}

\begin{abstract}
The combined effect of pressure and temperature on the mechanical behavior of polyurea is characterized and integrated into a constitutive model based on Williams-Landel-Ferry (WLF) equation. An increase in pressure is related to a decrease in temperature, both pressure and temperature affecting the polymer's molecular free volume and thereby introducing a pressure-dependent term into the WLF equation. The model is derived based on Fillers and Tschoegl's original work, regarding the free volume as a state variable. The model provides a closed-form expression for the time scale shift factor for equivalent temperature in terms of temperature and pressure and its threedimensional surface representation. The prediction of the present model agrees well with pressure shift factor data obtained from confined compression tests of polyurea, reported elsewhere, confirming our constitutive model and its experimental calibration procedure. A simplified model of the pressure effect is also developed which yields good approximate results.
\end{abstract}

Keywords: Modified WLF equation, time-temperature-pressure superposition, free volume

\footnotetext{
${ }^{*}$ Corresponding author

Email address: w.nantasetphong@gmail.com (W. Nantasetphong)
} 


\section{Introduction}

The relation between relaxation time and temperature in viscoelastic materials has been studied over the past 60 years. Many researchers have used the assumption of thermorheologically simple materials, which allows timetemperature superposition, to generate a master curve by shifting isothermal segments of relaxation tests, thereby extending the experimental time window beyond the normal range of a single test $[1,2,3,4]$. Many theories

for modeling the effect of temperature on the time-dependent behavior of polymers are based on the free volume concept. Doolittle and Doolittle [5] proposed this idea in their work on the viscosity of liquids. They found that the change in viscosity due to temperature might be expressed as a simple function of the net available volume between the polymer molecules, or free volume. William, Landel, and Ferry [4] extended this idea for application to polymers. They proposed that the fractional free volume, which is the ratio of free volume to total volume of a polymer, directly determines the mobility of the polymer chains, which in turn governs the time-dependent mechanical properties of the polymer. Above the glass transition temperature, $T_{g}$, the effect of temperature on the time-dependent mechanical properties is traditionally modeled by the well-known WLF equation, in which the fractional free volume is approximated by a linear function of temperature $[1,4]$. A more sophisticated free volume-based model was proposed by Knauss and Emri [6, 7]. Their model is able to predict nonlinear response of several polymers. They suggested that the fractional free volume should depend on the temperature, mechanical dilatation and swelling histories. In the case of negligible moisture and dilatational effects their model reduces to the WLF equation. Popelar and Liechti modified this model to include a distortional effect by introducing a new term, similar to the dilatational one [8, 9]. Losi and Knauss modified the free volume-based model for temperature below $T_{g}$ [10]. Other than the fractional free volume concept, the effect of temperature can be modeled via other thermodynamic quantities, e.g. the excess configurational entropy [11, 12], the excess enthalpy [13], and the relaxation or retardation process as the physical counterpart of a chemical reaction [14].

While the effect of temperature on the viscoelastic properties of polymers is fairly well understood, relatively little information is available on the effect of pressure. Generally, volume is reduced as temperature is decreased and/or pressure is increased. One could equate the effect of increasing pressure to that of decreasing temperature following this rationale. Ferry and Strat- 
ton assumed that the fractional free volume decreases linearly with pressure, yielding an equation of shift factor that has the same form as the WLF equation [15]. O'Reilly assumed that the fractional free volume is a function of the inverse of pressure, obtaining a simple exponential form for the shift factor [16]. However, it has been found that the dependence of the empirical shift distances on pressure could not be successfully described by either the FerryStratton or the O'Reilly equation [17]. The present work shows that these two models are not appropriate for the behavior of polyurea in particular. Fillers and Tschoegl proposed that pressure is associated with the change in fractional free volume via compressibility, which is defined as the inverse of the tangential bulk modulus $[17,18]$. They considered the fractional free volume to be a state variable depending on pressure and temperature and developed an equation for shift factor, which describes the pressure dependence and contains the WLF equation as a limiting case. They showed that shifting isobaric segments of mechanical properties of viscoelastic materials could yield the similar master curves as shifting the isothermal segments. The materials that allow isobaric shifting are referred to as piezorheologically simple materials.

The change in fractional free volume due to pressure is related to compressibility of the free volume, and the change due to temperature is associated with thermal expansivity. With the assumption of the fractional free volume being a state variable, its differential may be written accordingly as the sum of the two effects [17]. To consider the total change with pressure and temperature, Fillers and Tschoegl proposed two different integration paths; (1) integrate the pressure-dependent term at a fixed temperature first, followed by the temperature-dependent term at a fixed pressure, and (2) integrate the temperature-dependent term at a fixed pressure first, followed by the pressure-dependent term at a fixed temperature. They suggested that the first path is preferable since the first path requires knowledge of the pressure dependence of the expansivity while the second requires knowledge of the temperature dependence of the compressibility that is more difficult to determine. However, the second integration path yields the WLF equation as a limiting case without having to change its constants for each reference pressure. Moreover, one does not have to complete the stress relaxation test under pressure to obtain pressure-dependent parameters, as it is required with the first integration path. In this work, we extend Fillers and Tschoegl's work, utilizing the second integration path and designing the experimental framework to extract all desired constitutive parameters. For the sake of 
clarity in presentation, we will refer to this model as modified WLF equation (mWLF). However the original authors call it, the FMT model.

Polyurea, a block copolymer formed from the chemical reaction of diisocyanates with polyamines, was studied under high-pressure using ultrasonic measurements. The test specimens were quasi-statically compressed in confined uniaxial deformation. The longitudinal ultrasonic wave speed in the sample was measured at various pressures and temperatures. Then, the longitudinal moduli at various temperatures and pressures were calculated, using the wave speeds and densities of the samples, modified by the uniaxial strain. The testing pressure and temperature ranges in this study are 0.1 to $900 \mathrm{MPa}$ and 223 to $303 \mathrm{~K}$. The longitudinal modulus was used to relate the increase in pressure to the decrease in temperature at ambient pressure that would have yielded the same longitudinal modulus. The mWLF model gives the necessary shifts along the time (or frequency) axis that would lead to overlapping and alignment of the measured isothermal and isobaric viscoelastic modulus curves. The shift factor may be considered as the combination of two parts that account for effects of temperature and pressure. All parameters controlling the time scale shifts needed for the effect of temperature may be obtained from the original WLF equation. Furthermore, one can construct a function that for a given applied pressure gives the equivalent reduction in temperature, which induces the similar change in material behavior as the applied pressure. All remaining parameters for the effect of pressure can be found by curve-fitting this function. The mWLF model can be presented by a three-dimensional surface of the necessary shift in time or frequency scale as a function of temperature and pressure. The model fits very well with the present experimental data. Moreover, when specialized to the appropriate experimental range (0-900 MPa and 223-303 K) the predictions of the model also agree quite well with the previous study on nonlinear viscoelastic behavior of polyurea, conducted by Chevellard et al [19].

\section{Experiment}

\subsection{Material}

Polyurea (PU) is a block copolymer formed from a chemical reaction of an aromatic isocyanate component (hard segment) and an amine-terminated resin blend component (soft segment). The aromatic isocyanate component, commercial name Isonate 143L, is manufactured by Dow Chemicals [20]. It is a polycarbodiimide-modified diphenylmethane diisocyanate. It is in liquid 
form at room temperature and has a low viscosity and good storage stability down to $297 \mathrm{~K}\left(24^{\circ} \mathrm{C}\right)$. The amine-terminated resin blend component, commercial name Versalink P-1000, is an oligomeric diamine manufactured by Air Products [21]. It is a polytetramethyleneoxide-di-p-aminobenzoate and is liquid at room temperature, which allows it to be mixed, cast, and cured in ambient conditions. The reaction between Isonate 143L and Versalink P-1000 is generally very fast and insensitive to humidity and low temperatures. Theoretically, Isonate 143L and Versalink P-1000 should be mixed in a stoichiometric ratio of $1: 1$, i.e., the total number of isocyanate groups must equal the total number of amine groups in order to obtain complete chemical reaction. Holzworth et al. also showed that the stoichiometric ratio affects the mechanical properties of polyurea [22]. However, a slight $5 \%$ excess of Isonate $143 \mathrm{~L}$ is typically recommended by the manufacturer to ensure that the reaction is completed and to create a lightly cross-linked polymer [21]. It is worth noting that this small extra portion of Isonate does not introduce inhomogeneity into the polyurea system, but it helps to compensate for the amount that is lost upon reacting with residual moisture during storage or application, and therefore it is not expected to change the viscoelastic behavior or relaxation mechanism of polyurea significantly [22].

\subsection{Sample Preparation}

Samples were fabricated according to the process described in [22]. Teflon molds were used to cast test specimens with the geometry shown in Figure 1b. This geometry was selected to match with the high-pressure cell described below. The polyurea specimens were cured for 2 weeks at room temperature in a humidity-controlled environmental chamber that maintains $10 \%$ relative humidity. Twelve specimens were fabricated in one batch.

\subsection{Experimental Setup}

The test system consists of ultrasonic wave measurement setup, quasistatic confined loading setup, and temperature control chamber. The ultrasonic measurements were performed in the through-transmission mode, using a desktop computer with a Matec TB-1000 Toneburst card, two Panametrics videoscan longitudinal transducers (V103 Panametrics-NDT OLYMPUS), a 100:1 attenuator box, and a digital oscilloscope (Tektronix DPO3014) [23]. Toneburst signals of prescribed frequencies were generated from the card and split through BM-174-3 cables to the pitching transducer and the attenuator box, where the voltage was reduced by a factor of 100 to be measured on the 
scope. The received signal at the catching transducer was sent directly to the scope where the amplitude and travel time were measured.

Instron servo-hydraulic machine model 1332 was used as loading test machine. It has the capability to generate forces up to $100 \mathrm{kN}$. The compression mode was used in this study. MTS controller model 407 was used to control the force and displacement. Either displacement or force control can be selected. The loading rate is adjustable depending on the test condition. In this study, the force control mode was used with the slow loading rate of $67 \mathrm{~N} \mathrm{~s}^{-1}$ to ensure that sample was under quasi-static condition. An MTS environmental chamber model 651 was used to provide a cooling range from ambient to $144 \mathrm{~K}$ using liquid nitrogen. Testing temperature was controlled by MTS temperature controller model 409.80 .

To create quasi-hydrostatic conditions for the sample, a confinement cell was made of maraging steel (see Figure 1). The nearly cylindrical geometry and small size were chosen to achieve high pressure of $1000 \mathrm{MPa}$ at the maximum force load of $100 \mathrm{kN}$, while avoiding failure. The cell structure has no sharp edge, thereby minimizing stress concentration. Two vertical flat surfaces were machined on the two opposite sides of the cell where the two transducers were placed. Two pistons with the same cross-sectional shape of the sample were used for loading, while inside the cell, two filler columns were aligned to ensure that the sample surfaces were flat and parallel to the surfaces of the two transducers (see Figure 1). Screws, nuts, springs, fasteners, and cover plates were used to hold the test setup and to provide the constant applied load on the two transducers.

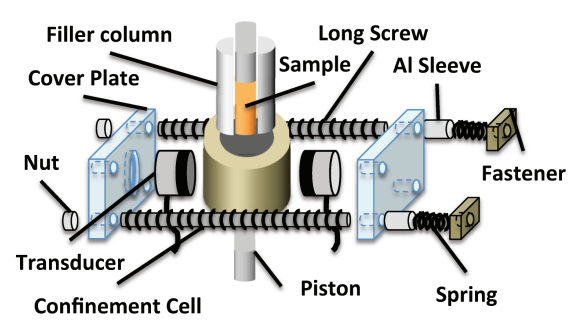

(a)

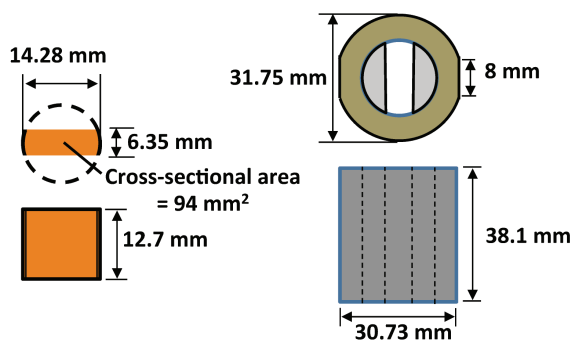

(b)

(c)

Figure 1: (a) Components of high-pressure test fixture (b) Sample (c) Confinement cell 


\subsection{Experimental Procedure}

The confinement cell calibration process was done at ambient condition. The special shape of the cell leads to the complexity of the wave speed measurement; internal reflections and interferences. The measured transmission can be roughly divided into two components (see Figure 2): traveling in a direct path through the sample and traveling around the cell body. The purpose of the cell calibration is to eliminate the effect of the second component from the measurement of time of travel in the cell $\left(t_{C}\right)$. In calibration test 1, we filled the cell with water, sent ultrasonic wave through the cell, and recorded the input and the received signals (see Figure 3a). We used water because the wave speed in water at room temperature was known $\left(1484 \mathrm{~m} \mathrm{~s}^{-1}\right)$, and it could completely fill the cell without gaps. The received signal in test 1 consisted of both the direct-path and go-around components added (see Figure 2). In test 2, we repeated this for an empty cell. The received signal in test 2 is only the go-around component. Subtracting the received signal in test 2 from the received signal from test 1, the direct-path signal was obtained (see Figure $3 \mathrm{~b}$ ). Note that since the impedance mismatch between steel and water is very large, the effect of transmission into the water on the go-around signal is neglected. Furthermore, since we are only interested in the timing of the pulse, a small error in the amplitudes due to this assumption is not expected to affect the eventual results. Finally, the consistency of the shape and amplitude of the go-around signals in Figure 3a, fortifies the relevance of this assumption (the input pulses are the same in both tests). We estimated the total time of travel in the direct path $\left(t_{T}\right)$ using the first peaks of the input and the direct-path signals (see Figure $3 \mathrm{~b}$ ). We calculated the time of travel in the water $\left(t_{W}\right)$, using the known wave speed and gap width of $6.35 \mathrm{~mm}$. The time of travel in the cell $\left(t_{C}\right)$ can be found by subtracting $t_{W}$ from $t_{T}$ (see Figure 2). Since the cell is made

of maraging steel, whose mechanical properties do not change much in the range of testing temperature and pressure, we assume that $t_{C}$ is constant. This agreed with our observations on the wave speed in the steel as we varied the temperature. We found that $t_{C}$ equals $4.564 \mu \mathrm{s}$ at $1 \mathrm{MHz}$. It will be used later in the wave speed measurement. Note that $t_{C}$ can also be estimated by dividing the total thickness of the steel in the direct-path propagation with the known wave speed in the steel $\left(5490 \mathrm{~m} \mathrm{~s}^{-1}\right)$. From this method, $t_{C}$ is $4.442 \mu \mathrm{s}$ which is in very close agreement with the value above.

After the cell calibration was finished, the wave speed measurement was performed. The testing temperature ranges from 223 to $303 \mathrm{~K}$ with $10 \mathrm{~K}$ 


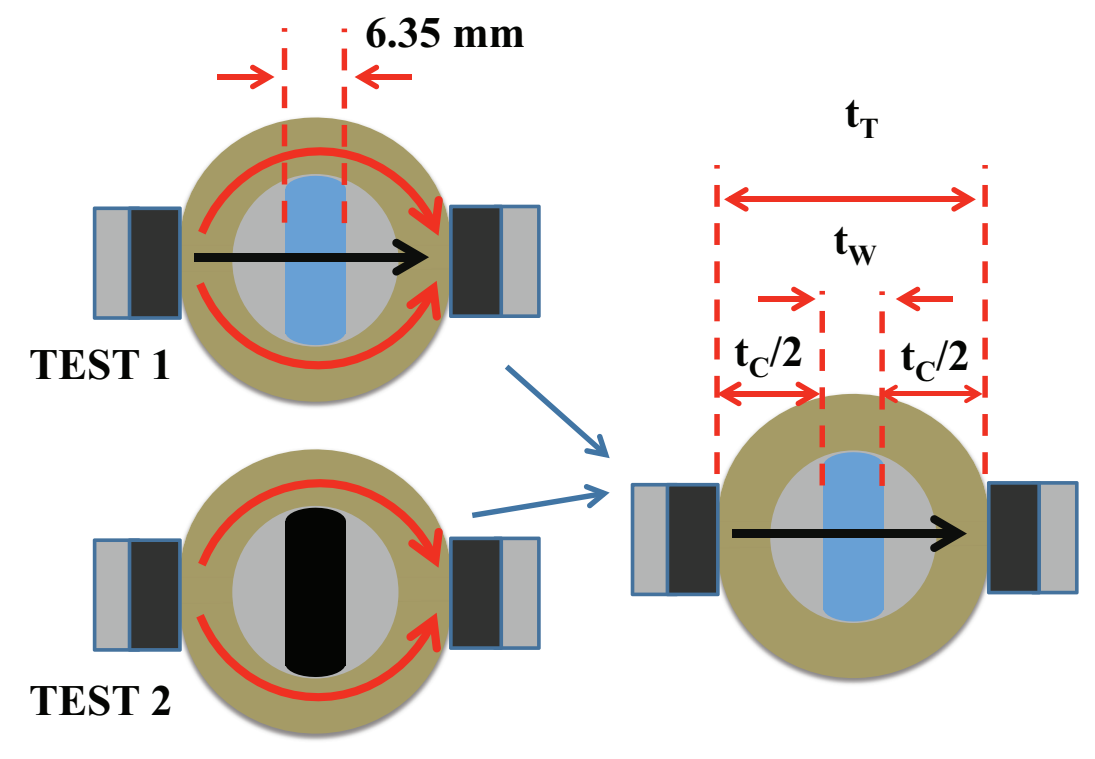

Figure 2: Confinement cell calibration; $t_{T}$ is total time of travel of direct-path signal, $t_{C}$ is half of time of travel in the cell, $t_{W}$ is time of travel in the water. The gap thickness is $6.35 \mathrm{~mm}$

steps. At each testing temperature, the sample was loaded from ambient pressure to $900 \mathrm{MPa}$. After loading, the sample could not be reused because it was deformed and lost its initial shape (though the material is highly elastic in compression, at very high pressures it will extrude in shear through the very small gaps between the pistons and the cell body). Nine samples, all made in the same batch, were used for the nine testing temperatures. Before testing, dimensions and densities of all samples were measured. Longitudinal wave couplant was applied over all surfaces of the samples and where the cell touched the samples and the transducers. To measure the wave speed in the sample, two measurements were required at each testing temperature. In test 1 , the sample was inserted into the confinement cell and capped with pistons from top and bottom (see Figure 1a). The two transducers were placed at the middle of the flat surfaces of the cell and locked in position with the two cover plates. In order to maintain relatively constant contact pressure between the transducers and the cell, two identical springs, fasteners and aluminum columns were employed together. The whole test fixture was held together by spring force (see Figure 1a). The test fixture was then placed 

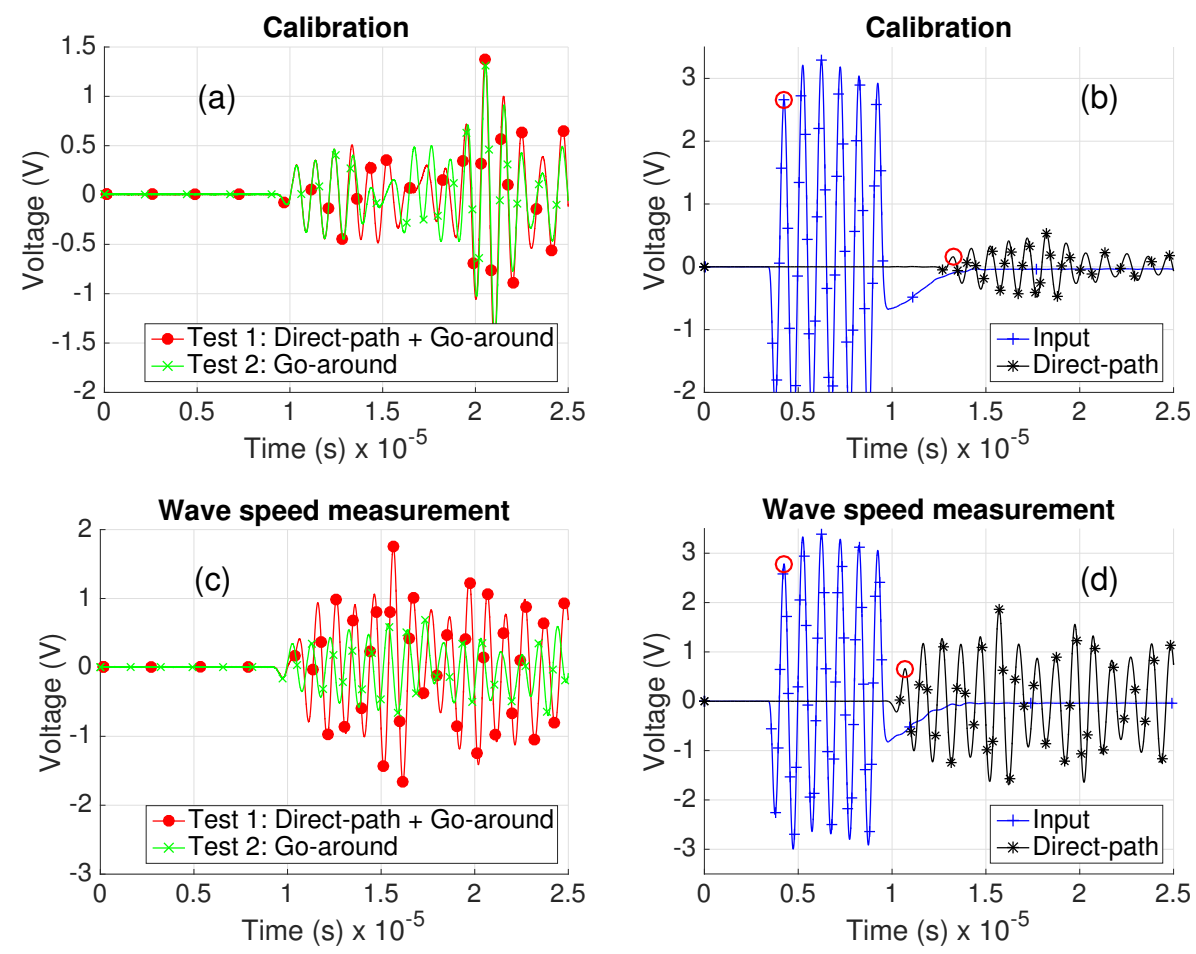

Figure 3: (a) Received signals from test 1 and 2 in cell calibration. (b) Total time of travel in the direct-path $\left(t_{T}\right)$ of the calibration is equal to the time difference between the two red circles. (c) Received signals from test 1 and 2 in wave speed measurement. (d) Total time of travel in the direct-path $\left(t_{T}\right)$ of the wave speed measurement is equal to the time difference between the two red circles. Note: The direct-path signal in (b) and (d) are obtained by subtracting the Go-around from the Direct-path + Go-around, in (a) and (c). The input signal is the same for all tests.

in an environmental chamber and the whole test fixture was equilibrated at the testing temperature for an hour, before the compressive load was applied on the sample at the loading rate of $67 \mathrm{~N} \mathrm{~s}^{-1}$. The polyurea sample in the cell was under quasi-hydrostatic conditions due to the slow loading rate and nearly incompressible nature of the elastomer. The testing pressure range is from 25 to $900 \mathrm{MPa}$, with $25 \mathrm{MPa}$ increments up to $400 \mathrm{MPa}$ and $50 \mathrm{MPa}$ increments for the rest. At each pressure step, the loading machine was stopped and held at constant load, while the ultrasonic measurement was performed at nominal frequency $1 \mathrm{MHz}$, and data were recorded. The compressive force and axial displacement histories were measured by the load cell and linear variable differential transformer (LVDT), respectively. They 
were recorded in a Labview signal express text file for analysis. In test 2, the test fixture was assembled empty and was equilibrated in the environmental chamber at the same temperature as test 1 for an hour. No pressure was applied on test fixture, though the springs were tightened to the same level as test 1. After 1-hour equilibration, the signal was sent through the empty confinement cell to record the go-around signal for the given temperature. Example of recorded signals test 1 and test 2 from wave speed measurement are shown in Figure 3c.

Subtracting the received signal in test 2 (without sample) from the received signal from test 1 (with sample), the direct-path signal was obtained (see Figure 3d). The total time of travel in the direct path $\left(t_{T}\right)$ was measured using the first peaks of the input and the direct-path signals (see Figure 3d). Subtracting $t_{C}$ previously obtained from cell calibration process from $t_{T}$, the time of travel in the sample $\left(t_{S}\right)$ at the testing temperature and pressure was obtained. We then repeated the process of finding $t_{S}$ for other testing temperatures and pressures. Longitudinal wave speed at each testing temperature and pressure can be calculated as:

$$
v_{L}(T, P)=\frac{d_{g}}{t_{S}(T, P)},
$$

where the constant $d_{g}=6.35 \mathrm{~mm}$ is the gap thickness shown in Figure 2, $t_{S}(T, P)$ and $v_{L}(T, P)$ are the time of travel in the sample and the longitudinal wave speed at testing temperature $T$ and pressure $P$, respectively. Longitudinal wave speed at each testing temperature and pressure is shown in Figure 4a. Uniaxial quasi-static stress and strain of the samples were also calculated, using the recorded force, displacement histories, and initial dimensions of the samples. Since polyurea is nearly incompressible material the confined compressive force creates quasi-hydrostatic pressure on the sample. Machine stiffness was also measured for correcting the measured strain in the sample.

$$
\begin{gathered}
\sigma=P=\frac{F(t)}{A}, \\
\epsilon(T, P)=\frac{d(T, P)-d\left(T, P_{o}\right)}{h},
\end{gathered}
$$

where $\sigma$ is engineering stress (or pressure $(P)$ in this particular case), $\epsilon(T, P)$ is compressive engineering strain at $T$ and $P, F(t)$ is compressive force history, $d(T, P)$, and $d\left(T, P_{o}\right)$ are displacements at $T$ and $P$, and $T$ and $P_{o}$, 
respectively. $h$ and $A$ are the initial height and cross sectional area of the sample (see Figure 1b). The stress and strain are shown in Figure 4b. The slope of the stress-strain plot yields the tangential quasi-static longitudinal modulus. With the compressive strain at each temperature and pressure, polyurea density, $\rho(T, P)$, can be calculated as follows:

$$
\rho(T, P)=\frac{\rho_{o}}{\left(1+3 C_{T E}\left(T-T_{o}\right)+\epsilon(T, P)\right)},
$$

where $\rho_{o}$ is the density of polyurea at room temperature $T_{o}$ and ambient pressure, and $C_{T E}$ is the thermal expansion coefficient of polyurea $\left(207 \mu \mathrm{m} \mathrm{m}^{-1} \mathrm{~K}^{-1}\right)$. $\rho(T, P)$ is shown in Figure 4c. The $1 \mathrm{MHz}$ dynamic longitudinal modulus at each temperature and pressure can be calculated as:

$$
L(T, P)=\rho(T, P)\left[v_{L}(T, P)\right]^{2},
$$

where $L(T, P)$ is the dynamic longitudinal modulus at $1 \mathrm{MHz}$, temperature $T$, and pressure $P . L(T, P)$ is shown in Figure 5 .

\subsection{Experimental results}

During experiment, the samples were loaded slowly and step-wise from atmospheric pressure all the way to the maximum value of $900 \mathrm{MPa}$, and the ultrasonic data were collected at each pressure of interest. The unloading data were not considered here. The measured longitudinal wave speed is shown in Figure 4a. The wave speed at each temperature can be divided into two segments, with the first having a higher slope than the second. It is hypothesized that the bend in each line represents a transition in the material from rubber to glassy. As temperature decreases the glass transition point shifts toward lower pressure. At higher temperatures the transition point is more pronounced; while, at lower temperatures it exhibits a more gradual curve-like behavior. Figure $4 \mathrm{~b}$ shows the compressive stress (to be an estimate of the pressure)-compressive strain plots. At $223 \mathrm{~K}$ polyurea undergoes $11 \%$ strain at $900 \mathrm{MPa}$; whereas, at $303 \mathrm{~K}$ it takes $16 \%$ strain. The quasi-static longitudinal modulus can be obtained from the slope of the stress-strain plot. However it is not clean due to the effect of the noise in the strain measurement, therefore it is not shown here. Figure 4c shows the density of polyurea versus pressure. When pressure is above $300 \mathrm{MPa}$, the density measured at higher temperatures shows a higher slope, and all the curves converge to around $1200 \mathrm{~kg} \mathrm{~m}^{-3}$ at $900 \mathrm{MPa}$. 
Figure 5 shows the ultrasonic longitudinal modulus $(L)$ at $1 \mathrm{MHz}$. It increases with increasing pressure and decreasing temperature. Similar to longitudinal wave speed, we can somewhat see the ultrasonic longitudinal modulus has two distinct segments; higher slope at the first segment and smaller slope at the second segment. However, due to the effect of density in longitudinal modulus (see equation 5), this behavior is less pronounced. Despite the less apparent glass transition bend point, its location is the same as in the longitudinal wave speed. Paterson did measurement of Young's moduli of polyureathane, as a function of pressure up to $1000 \mathrm{MPa}$ at $293 \mathrm{~K}$ $\left(20^{\circ} \mathrm{C}\right)$ and found similar behavior [24]. In Paterson's work, the glass transition of polyureathane occurs at around $400 \mathrm{MPa}$. Here the glass transition of polyurea happens at around $350 \mathrm{MPa}$ at the same temperature. Paterson did not determine the glass transition temperature of polyurethane at atmospheric pressure; however, he provided the number from the supplier, $205 \mathrm{~K}\left(-68^{\circ} \mathrm{C}\right)$. For our polyurea sample, the glass transition is found to be around 213 to $223 \mathrm{~K}\left(-60\right.$ to $\left.-50^{\circ} \mathrm{C}\right)$ [25]. At each temperature, the glass transition occurs when the $1 \mathrm{MHz}$ dynamic longitudinal modulus is higher than $8 \mathrm{GPa}$. Note that at lower temperatures, the glass transition point shifts toward lower pressure. Paterson did not test at other temperatures.

\subsection{Analysis of the experimental data}

In order to use the data of the longitudinal modulus for time-temperaturepressure superposition, a slight modification is needed. The $1 \mathrm{MHz}$ longitudinal modulus at each temperature and pressure, $L(\omega, T, P)$ needs to be normalized, by a factor that depends theoretically on the density and temperature (vertical shift), based on the flexible chain theory $[1,18]$.

$$
L_{R}(\omega, T, P)=L(\omega, T, P) \frac{\rho_{r e f} T_{r e f}}{\rho T},
$$

where $L_{R}(\omega, T, P)$ is the reduced longitudinal modulus. $\omega$ is the angular frequency. $\rho_{\text {ref }}$ is the density of polyurea at reference temperature, $T_{r e f}$, and

pressure, $P_{\text {ref }}$. For the following analysis, $\rho_{\text {ref }}$ and $T_{\text {ref }}$ can be arbitrary chosen since they are constant multipliers; so we use 1 for both of them. Figure 6a shows $L_{R}$ as a function of pressure at each testing temperature.

Following Fillers and Tschoegl [17], the fractional free volume is considered a state variable that depends on pressure and temperature. Instead of dealing with both pressure and temperature separately, we seek to find 

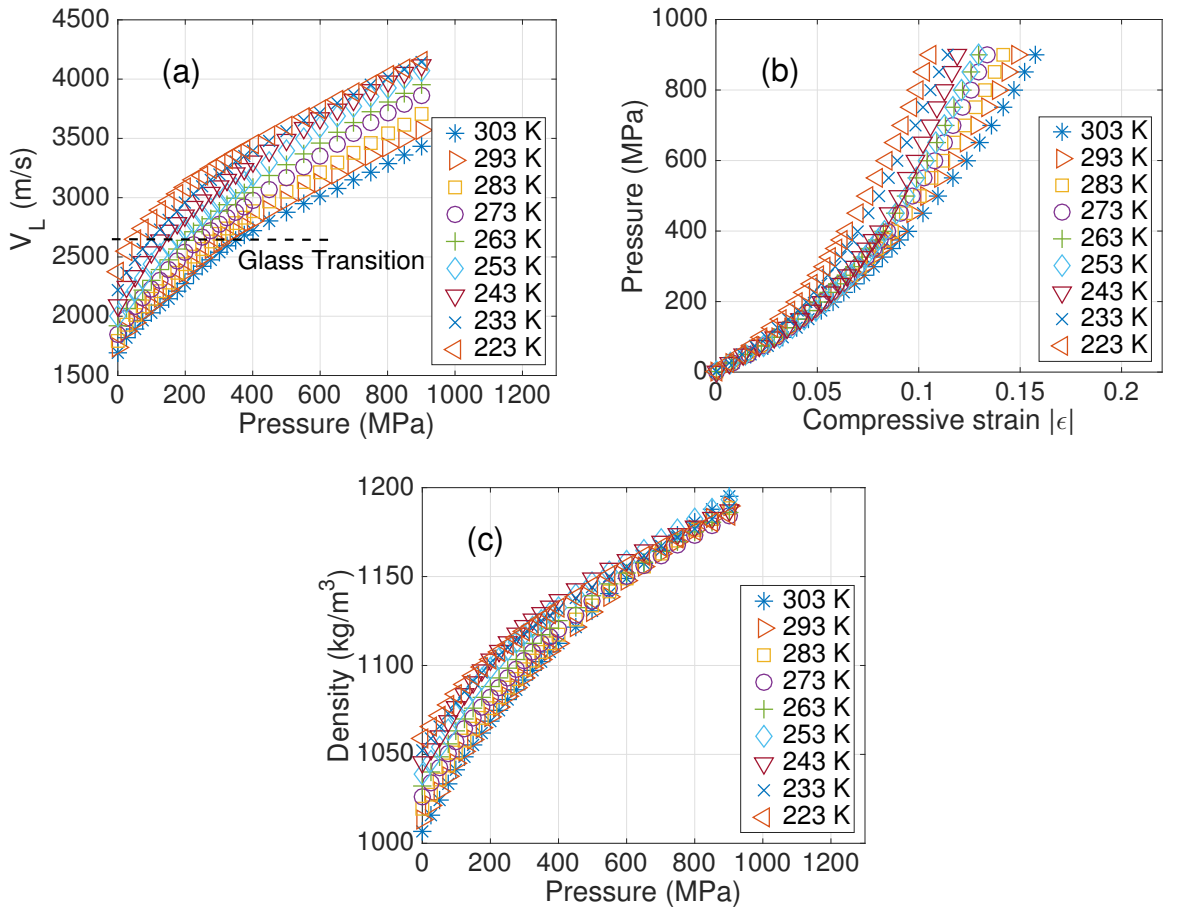

Figure 4: (a) Longitudinal wave speed, $v_{L}$ versus pressure. (b) Pressure versus compressive strain. (c) Density of polyurea versus pressure.

a relation between pressure and temperature that yields the same state of the fractional free volume. It is worth noting that the fractional free volume is not calculated directly in our study. But it is associated to the reduced longitudinal modulus. If two different states of polyurea with different temperatures and pressures yield the same reduced longitudinal modulus, they are assumed to have the same fractional free volume. To do this, the following process is used:

1.Consider only data point below glass transition pressure, above which the time-temperature-pressure superposition cannot be applied (see Figure 6a). Glass transition temperature of polyurea is around 213-223 K at atmospheric pressure [19, 25].

2.Consider data of a testing temperature and use interpolation to relate pressure to temperature that yields the same $L_{R}$ (see Figure 6b). Collect pressure-temperature pairs.

3.Repeat the process for other testing temperatures. 


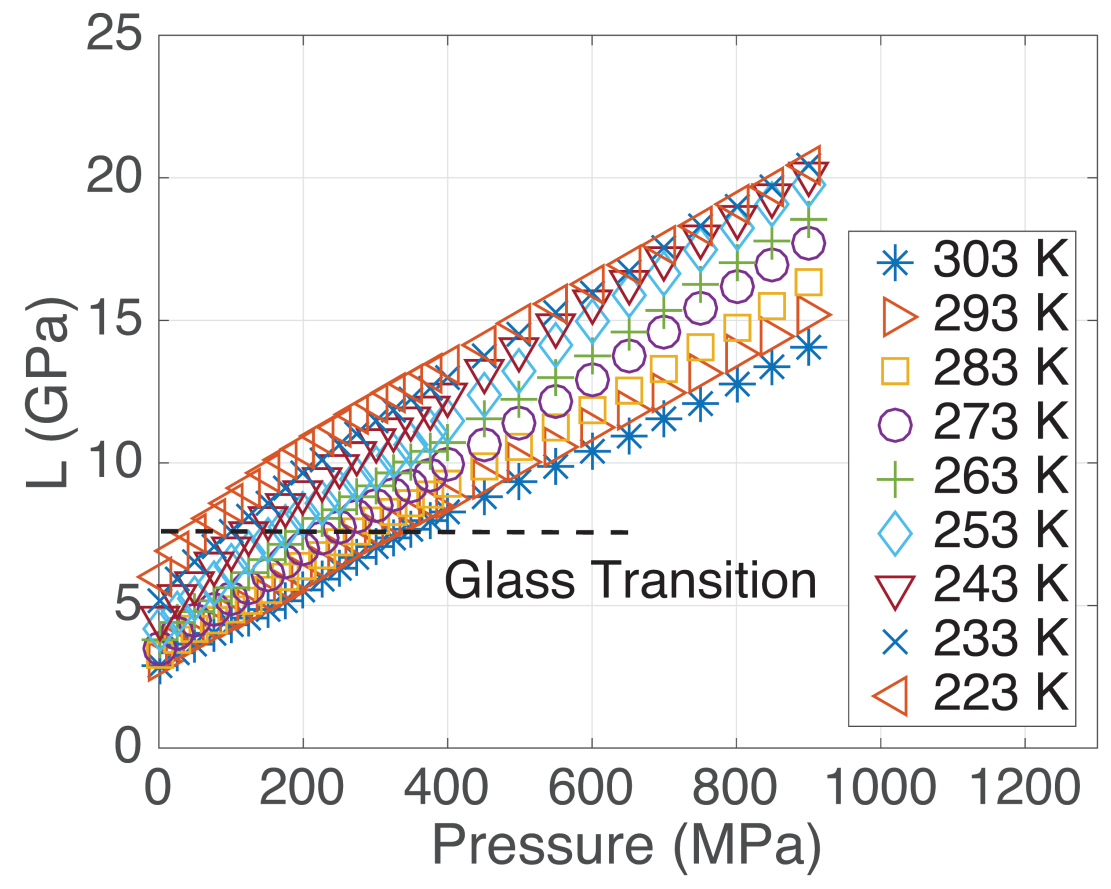

Figure 5: Dynamic longitudinal modulus, $L$ at $1 \mathrm{MHz}$ versus pressure.

Figure $6 \mathrm{c}$ shows the relation between pressure and temperature that give the same value of the reduced dynamic longitudinal modulus $L_{R}$. Each line represents each testing temperature and maps the testing pressure to the corresponding testing temperature at $0.1 \mathrm{MPa}$ (ambient pressure) that has the same $L_{R}$. Figure $6 \mathrm{~d}$ relates the increasing in pressure to the amount of temperature reduction, depending on the initial temperature. Each line has different number of data points since the minimum temperature in the interpolation process is always $T_{g}=223 \mathrm{~K}$. We remark that the reduction in temperature that is resulted from pressure may be different if we use longitudinal modulus at other frequencies. However, in the absence of any information on this point we assume that the difference is negligible.

\section{Discussion}

Figure 6a shows a point or a very narrow range for each testing temperature, where the slope of the reduced longitudinal modulus changes. At these transition pressures, the behavior of polyurea changes from rubber-like 

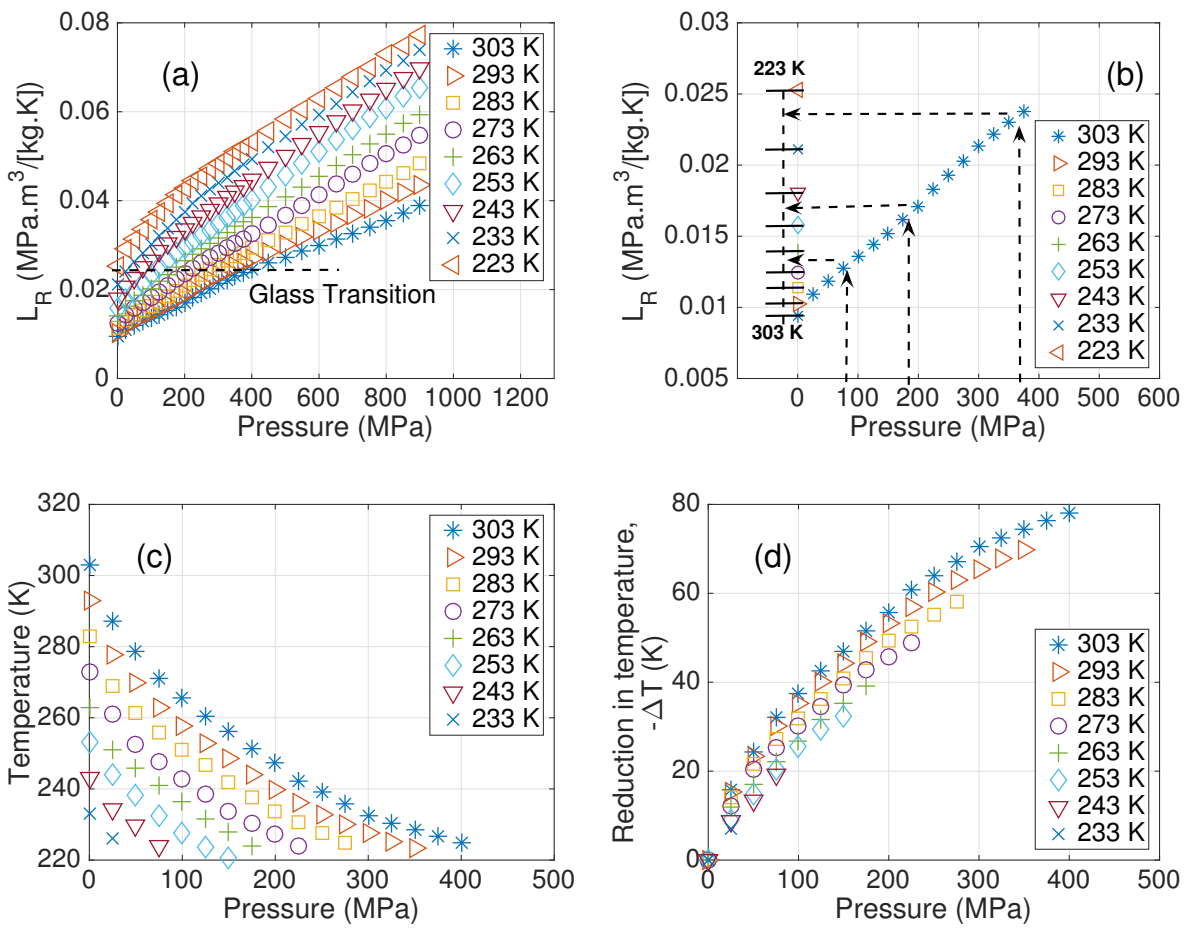

Figure 6: (a) The reduced dynamic longitudinal modulus, $L_{R}$ versus pressure and temperature (b) Interpolation to find relation between temperature and pressure (c) The apparent temperature for a given actual temperature and applied pressure (d) Reduction in temperature versus pressure for a given actual temperature

to glass-like. We limit the analysis in this paper to the rubbery range to be compatible with the free volume-based WLF approach. It can be seen in Figure $6 c$ that the equivalent temperature is more sensitive at lower pressure values, suggesting that it might asymptotically approach to a limit value at high pressures. However, the material becomes glassy before that state is reached. Figure 6d supports this conclusion. If all the curves in this figure were to collapse on to a common one, a much simpler formulation would have been possible. In what follows, we will implement Fillers and Tschoegl's idea to derive the modified WLF equation (mWLF) for a general case of the combined temperature and pressure effects [17].

\subsection{Theory}

The main idea of the modification of the WLF equation in this work follows that of Fillers and Tschoegl $[17,18]$. However, we proposed an al- 
ternative material testing method compatible with the mWLF model and that is less time-consuming for nearly incompressible viscoelastic materials. Since the fundamental derivation of the model was explained thoroughly in the original papers, only the important modification will be discussed here.

The shift factor, $a_{T P}$, is a function of fractional free volume defined as the ratio of the free volume inside a polymer to the total volume (free volume plus occupied volume) $[1,4]$. It is expressed as,

$$
\log a_{T P}=\frac{B}{2.303}\left(\frac{1}{f(T, P)}-\frac{1}{f\left(T_{o}, P_{o}\right)}\right),
$$

where the factor $B$ is a constant, $f(T, P)$ is the fractional free volume at temperature $T$ and pressure $P, f\left(T_{o}, P_{o}\right)$ is the fractional free volume at reference temperature $T_{o}$ and pressure $P_{o}$. The fractional free volume $f(T, P)$ is assumed to be conservative. The change of $f(T, P)$ does not depend on path. The differential of $f(T, P)$ or the infinitesimal change of $f(T, P)$ can be written as follows:

$$
d f(T, P)=\left(\frac{\partial f}{\partial T}\right)_{P} d T+\left(\frac{\partial f}{\partial P}\right)_{T} d P .
$$

We integrate equation 8 from reference temperature $T_{o}$ and pressure $P_{o}$ to testing temperature $T$ and pressure $P$, using a path that first keeps pressure fixed at $P_{o}$, while increasing the temperature, followed by a segment that keeps the temperature fixed at $\mathrm{T}$ and increases the pressure:

$$
\int_{T_{o}, P_{o}}^{T, P} d f(T, P)=\int_{T_{o}}^{T}\left(\frac{\partial f}{\partial T}\right)_{P_{o}} d T+\int_{P_{o}}^{P}\left(\frac{\partial f}{\partial P}\right)_{T} d P .
$$

Fillers and Tschoegl preferred a different integration path [17]; they integrated $(\partial f / \partial P)_{T_{o}}$ from $P_{o}$ to $P$ and $(\partial f / \partial T)_{P}$ from $T_{o}$ to $T$. They chose to consider the pressure dependence of the expansivity of the free volume. In this work and consistent with the original WLF equation, only the expansivity of the free volume at ambient pressure is used. However, we have to deal with the temperature dependence of the compressibility (inverse of the bulk modulus) of the free volume. Moreover, this choice of path suits our experimental data more seamless. After working out equation 9 and substitution into equation 7 , the pressure-and-temperature dependent shift factor, $a_{T P}$ can be written as $[17,18]$ :

$$
\log a_{T P}=\frac{-c_{1}\left(T-T_{o}-\Omega(T, P)\right)}{c_{2}+T-T_{o}-\Omega(T, P)}
$$


where

$$
\begin{gathered}
c_{1}=\frac{B}{2.303 f\left(T_{o}, P_{o}\right)}, \\
c_{2}=\frac{f\left(T_{o}, P_{o}\right)}{\alpha_{f}\left(P_{o}\right)}, \\
\Omega(T, P)=c_{3}(T) \log \left(\frac{1+c_{4}(T) P}{1+c_{4}(T) P_{o}}\right)-c_{5}(T) \log \left(\frac{1+c_{6}(T) P}{1+c_{6}(T) P_{o}}\right), \\
c_{3}(T)=\frac{2.303}{\alpha_{f}\left(P_{o}\right) l(T)}, \\
c_{4}(T)=\frac{l(T)}{L\left(T, P_{o}\right)}, \\
c_{5}(T)=\frac{2.303}{\alpha_{f}\left(P_{o}\right) l_{\phi}(T)}, \\
c_{6}(T)=\frac{l_{\phi}(T)}{L_{\phi}\left(T, P_{o}\right)},
\end{gathered}
$$

$c_{1}$ and $c_{2}$ are constants in original WLF equation, $\alpha_{f}\left(P_{o}\right)$ is the expansivity of the free volume at $P_{o}, \Omega(T, P)$ is the additional pressure-dependent term that is introduced into the original WLF equation, parameters, $c_{3}(T)$, $c_{4}(T), c_{5}(T)$, and $c_{6}(T)$ are unknown parameters that depend on testing temperature $T, L\left(T, P_{o}\right)$ and $L_{\phi}\left(T, P_{o}\right)$ are the longitudinal moduli of the entire and occupied volumes respectively at $T$ and $P_{o}, l(T)$ and $l_{\phi}(T)$ are the derivatives of the longitudinal moduli of the entire and occupied volumes with respect to pressure, at temperature $T$, and the subscript $\phi$ on parameters indicates the relation to the occupied volume.

It is worth noting that $\Omega(T, P)$ in equation 13 is different from $\theta(P)$ in $[17,18]$. The form of equation 13 depends on two observations: (i) $\alpha_{f}\left(P_{0}\right)$ is kept constant at $P_{o}$ and independent of temperature and (ii) the bulk (or longitudinal) modulus of the polymer has a linear dependence on pressure with a slope that is a function of temperature only. This dependence is manifested in the coupling in the $\Omega(T, P)$ function. Considering that polyurea is nearly incompressible material, a modification was made here to replace the bulk modulus with the longitudinal modulus. In other words, for nearly incompressible polymers, e.g. polyurea, Poisson's ratio is around 0.4 (low temperature, glass-like behavior) - 0.5 (high temperature, rubber-like behavior). Thus the bulk modulus is significantly larger than the shear modulus 
and one may approximate the bulk modulus with the longitudinal modulus. For other polymers that are more compressible, this approximation might introduce some error, and therefore it should be used with caution. From our experimental observations, the longitudinal modulus of polyurea is a linear function of pressure up to the glass transition (see Figure 5), with a temperature-dependent slope and can be written as:

$$
L(T, P)=L\left(T, P_{o}\right)+l(T) P
$$

$L\left(T, P_{o}\right)$ and $l(T)$ can be obtained from fitting equation 18 to the experimental data below glass transition, which are shown in Figure 5. The results are shown in Figures 7a, 7b, and 7c. The longitudinal modulus of the occupied volume is very difficult to measure experimentally. It is assumed that it has the same form as the longitudinal modulus of the polymer [17, 18], i.e.

$$
L_{\phi}(T, P)=L_{\phi}\left(T, P_{o}\right)+l_{\phi}(T) P .
$$

$L_{\phi}(T, P), L_{\phi}\left(T, P_{o}\right)$, and $l_{\phi}(T)$ can be obtained using nonlinear regression analysis, after the final formulation is derived. It is worth mentioning that equation 10 represents the modied WLF equation that includes both effects of temperature and pressure. $\Omega(T, P)$ is the pressure dependent term introduced into the original WLF equation, representing extra apparent temperature reduction associated with pressure $P$. At $P=P_{o}, \Omega(T, P)=0$ and equation 10 reduces to the original WLF equation.

\subsection{Applications}

The function $\log a_{T P}$ in equation 10 is the temperature-pressure-dependent logarithmic time shift required to bring data recorded at the testing temperature $T$ and pressure $P$, into superposition with data recorded at the reference temperature $T_{o}$ and pressure $P_{o}$. In this study, $T_{o}$ and $P_{o}$ are $273 \mathrm{~K}$ and 0.1 MPa respectively. Materials that allow this superposition are called, thermorheologically and piezorheologically simple materials. Each polymer has different shift factor (though for temperature superposition, the WLF equation presents a very good uniform approximation formula for most polymers between $T_{g}$ and $\left.T_{g}+100 \mathrm{~K}\right)$ and can be characterized through the parameters: $c_{1}, c_{2}, c_{3}(T), c_{4}(T), c_{5}(T)$, and $c_{6}(T) . L\left(T, P_{o}\right)$ and $l(T)$, which appear in $c_{4}(T)$ can be obtained from fitting equation 18 to the experimental data below glass transition pressure that is shown in Figure 5. Figure 7a shows that the approximation and linearization in equation 18 agree very well with 
experimental data shown in Figure 5. $L\left(T, P_{o}\right)$ and $l(T)$ are shown in Figures $7 \mathrm{~b}$ and $7 \mathrm{c}$, considering only data in the range between 243 and $303 \mathrm{~K}$. We found that inverse of $l(T)$, which is a variable in parameter $c_{3}(T)$ is a linear function of temperature (see Figure $7 d$ ). It can be written in the following form:

$$
\frac{1}{l(T)}=I_{1}\left(T-I_{2}\right)
$$

where $I_{1}$ and $I_{2}$ can be found from linear curve fitting. They are shown in Table 1. With the constant $\alpha_{f}\left(P_{o}\right), c_{3}(T)$ can then be written in the following form:

$$
c_{3}(T)=c_{31}\left(T-c_{32}\right),
$$

where

$$
\begin{gathered}
c_{31}=\frac{2.303 I_{1}}{\alpha_{f}\left(P_{o}\right)}, \\
c_{32}=I_{2},
\end{gathered}
$$

$c_{31}$ is an unknown constant to-be-found based on parameter $\alpha_{f}\left(P_{o}\right) \cdot c_{4}(T)$ can now be calculated using equation 15 and is shown in Figure 7e. As we can see, $c_{4}(T)$ is independent of the temperature in the range from 243 to $303 \mathrm{~K}$. Therefore, we assume that $c_{4}(T)$ is constant and equal to the average of $c_{4}(T)$ :

$$
c_{4}(T)=\bar{c}_{4}(T)=c_{4} .
$$

$c_{4}$ is shown in Table1. Due to the lack of information about parameters $c_{5}(T)$, and $c_{6}(T)$, we assume that $c_{5}(T)$ also has a linear form similar to $c_{3}(T)$, and $c_{6}(T)$ is constant similar to $c_{4}(T)$. This is quite reasonable assumption due to the similar characteristics between $c_{3}(T)$ and $c_{5}(T)$, and $c_{4}(T)$ and $c_{6}(T)$ (see equations 14-17).

With equations 21 to 24 , and assumption that $c_{5}(T)$ and $c_{6}(T)$ are linear function of temperature and constant, respectively, a nonlinear regression analysis was performed using equation 13 and data in Figure 6d. The initial guess for $\alpha_{f}\left(P_{o}\right)$ was obtained using equations 11 and 12, with the assumption that parameter $B$ is 1 . Constants $c_{1}$ and $c_{2}$ were measured and recorded elsewhere [26]. They are shown in Table 1. The initial guesses for $c_{51}$ and $c_{52}$ were $c_{31}$ and $c_{32}$, respectively. Constant $c_{4}$ was used as the initial guess for $c_{6}$. The results of the regression analysis are shown in Figure 8a. Constants $c_{31}, c_{32}, c_{4}, c_{51}, c_{52}, c_{6}$, and $\alpha_{f}\left(P_{o}\right)$ are given in Table 1 . It is notable that $c_{32}$ and $c_{52}$ are very close to each other and are below the glass transition 

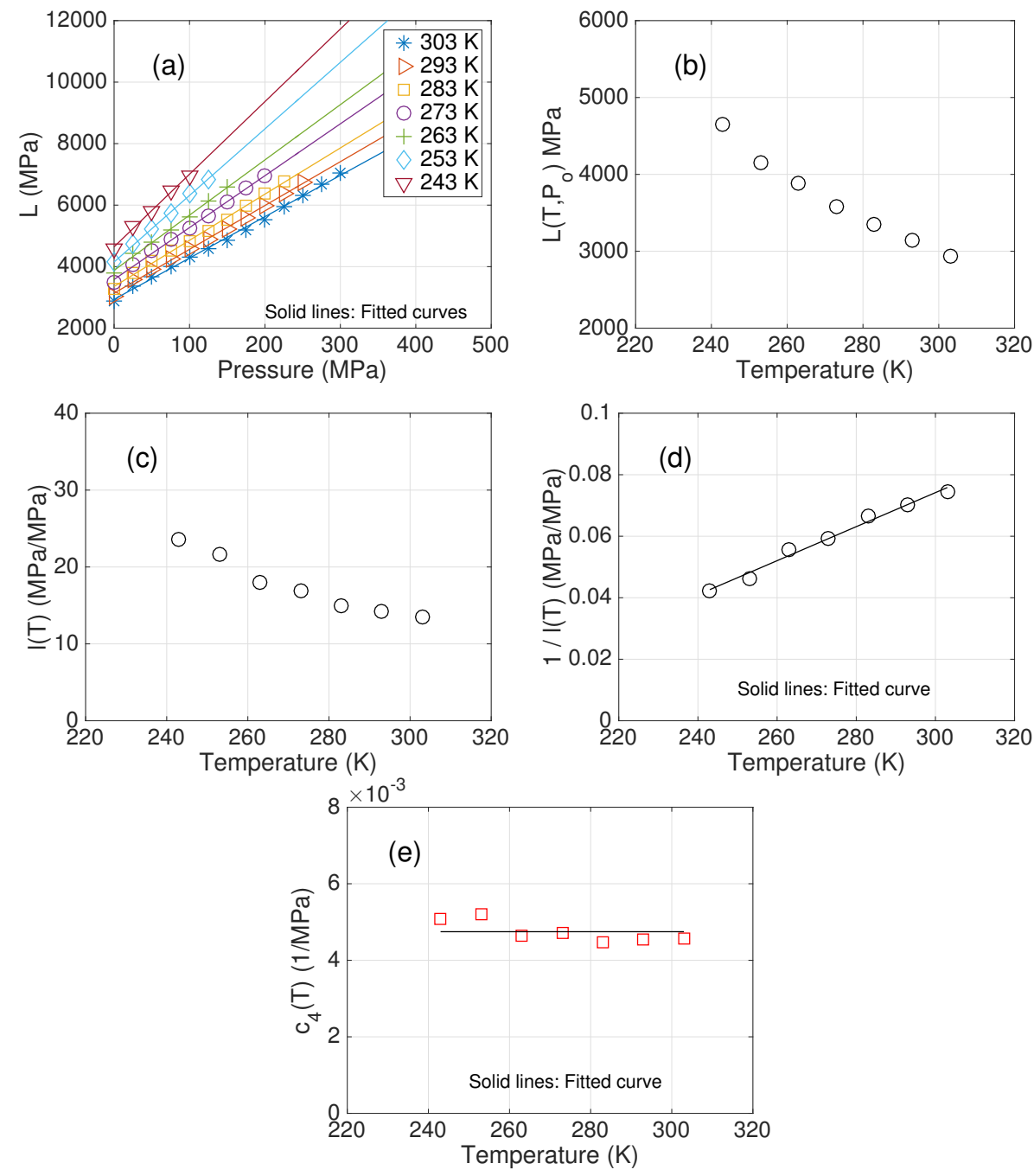

Figure 7: (a) Dynamic longitudinal modulus, $\mathrm{L}$ at $1 \mathrm{MHz}$ versus pressure. (b) Longitudinal moduli, $L\left(T, P_{o}\right)$ at $1 \mathrm{MHz}$ and atmospheric pressure versus temperature. (c) Slopes of longitudinal moduli, $l(T)$ at $1 \mathrm{MHz}$ versus temperature. (d) Inverse of $l(T)$ at $1 \mathrm{MHz}$ versus temperature. (e) $c_{4}(T)$ at $1 \mathrm{MHz}$ versus temperature

temperature of polyurea (213-223 K). As the temperature gets closer to these values, the effect of pressure on the shift factor diminishes. From the nonlinear regression analysis, we obtain all necessary parameters for $\Omega(T, P)$ in 
the mWLF equation which can now be written as:

$$
\Omega(T, P)=c_{31}\left(T-c_{32}\right) \log \left(\frac{1+c_{4} P}{1+c_{4} P_{o}}\right)-c_{51}\left(T-c_{52}\right) \log \left(\frac{1+c_{6} P}{1+c_{6} P_{o}}\right),
$$

Equations 10, and 25, with parameters shown in Table 1 yields the full form of the modified WLF equation that includes temperature and pressure effects for polyurea at reference temperature $(274 \mathrm{~K})$ and pressure $(0.1 \mathrm{MPa})$.
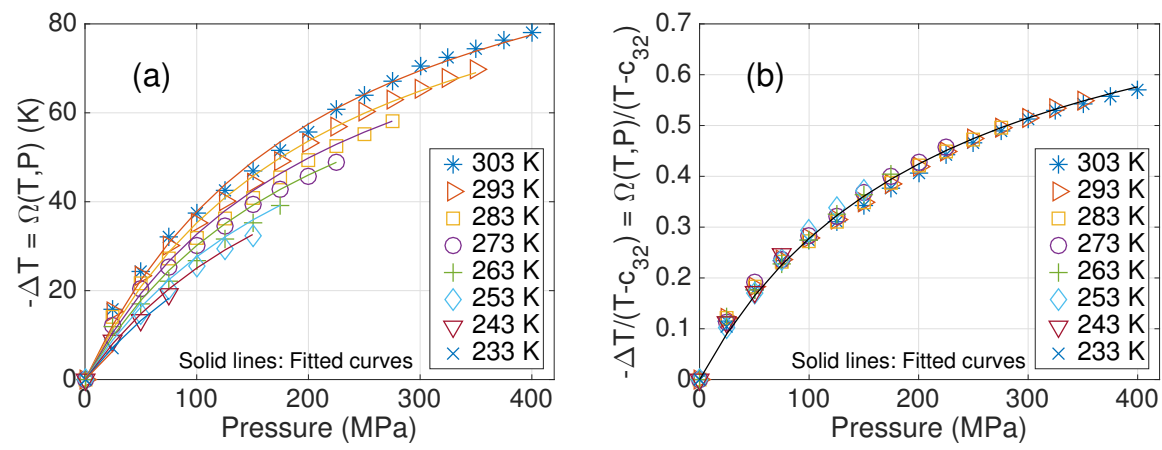

Figure 8: (a) Comparison between experimental data and the full model (equation 25) with parameters in Table 1. (b) Comparison between normalized experimental data and the normalized simplified model (equation 26) with parameters in Table 2.

Table 1: Parameters in the full form of the modified WLF equation for polyurea

\begin{tabular}{|c|c|c|c|}
\hline \multicolumn{4}{|c|}{ Polyurea } \\
\hline \hline$B$ & 0.839 & $c_{1}$ & 25.25 \\
\hline$f\left(T_{o}, P_{o}\right)$ & 0.0144 & $c_{2}(\mathrm{~K})$ & 208.9 \\
\hline$\alpha_{f}\left(P_{o}\right)\left(\mathrm{K}^{-1}\right)$ & $6.91 \mathrm{E}-05$ & $c_{31}$ & 18.45 \\
\hline$T_{o}(\mathrm{~K})$ & 274 & $c_{4}\left(\mathrm{MPa}^{-1}\right)$ & 0.00475 \\
\hline$P_{o}(\mathrm{MPa})$ & 0.1013 & $c_{51}$ & 18.65 \\
\hline$I_{1}\left(\mathrm{~K}^{-1}\right)$ & $5.53 \mathrm{E}-04$ & $c_{52}(\mathrm{~K})$ & 166.96 \\
\hline$I_{2}=c_{32}(\mathrm{~K})$ & 165.94 & $c_{6}\left(\mathrm{MPa}^{-1}\right)$ & 0.00423 \\
\hline
\end{tabular}

With $\alpha_{f}\left(P_{o}\right), c_{1}$, and $c_{2}$, the constants $B$, and $f\left(T_{o}, P_{o}\right)$ in the original WLF equation can be calculated, using equations 11 and 12 . These parameters are shown in Table 1 . It is interesting to note that the constant $B$ 
is not equal to 1 as commonly assumed. Clearly the ratio of $B / f\left(T_{o}, P_{o}\right)$ is the physically important quantity related directly to the horizontal shift function. At this point, we have all the required parameters for the modified WLF equation. The effect of pressure is added as an additional variable into the original WLF equation. The original WLF line in temperature plane now becomes a surface in temperature and pressure space. Figure 9a shows the three-dimensional plot of the shift factor of polyurea. The glass transition temperature of polyurea is around $223 \mathrm{~K}$ at ambient pressure. For other conditions, we assume that the value of shift factor is constant at glass transition state and calculate glass transition temperatures at elevated pressures, in agreement with Fillers and Tschoegl. Their experimental results showed that the glass transition, at which polymers in their tests becomes glassy, occurs at a constant value of $\log a_{T P}$ [17]. Note that on the isobaric graph, the shift factor of polyurea is concave upward with respect to temperature (see Figure 9b); while on the isothermal graph, the shift factor is concave downward with respect to pressure (see Figure 9c). In other words, as pressure increases, its effect on the isothermal shift factor becomes less pronounced. This observation is compatible with the fact that at very high pressures there is less free volume available for polymer chains; thus, increasing pressure further would not have as much effect as it does at low pressures. This behavior is also found in the previous study on nonlinear viscoelastic behavior of polyurea by Chevellard et al [19]. They performed confined compression tests to extract bulk and shear moduli. Their pressure shift factor (shown as crosses in Figure 10) was obtained from shifting isobaric bulk moduli at different pressures to form a bulk modulus master curve at $273 \mathrm{~K}$. They fitted the pressure shift factor with a quadratic equation (shown as dash-dotted line in Figure 10). The present model (shown as dashed line in Figure 10) reproduces their experimental data quite well, even though the present parameters were calibrated using longitudinal modulus data. In other words, the more efficient and less complicated process of measuring the longitudinal wave behavior under high pressure as described here could be utilized for a very good approximation of pressure effect. Furthermore, the present model does not use a separable formulation for the effects of pressure and temperature, therefore preserving the coupling between the two; see equation 13. Finally, Figure 9d shows $T_{g}$ as an increasing function of pressure, showing glassy response at room temperature under around $400 \mathrm{MPa}$ confining pressure. 

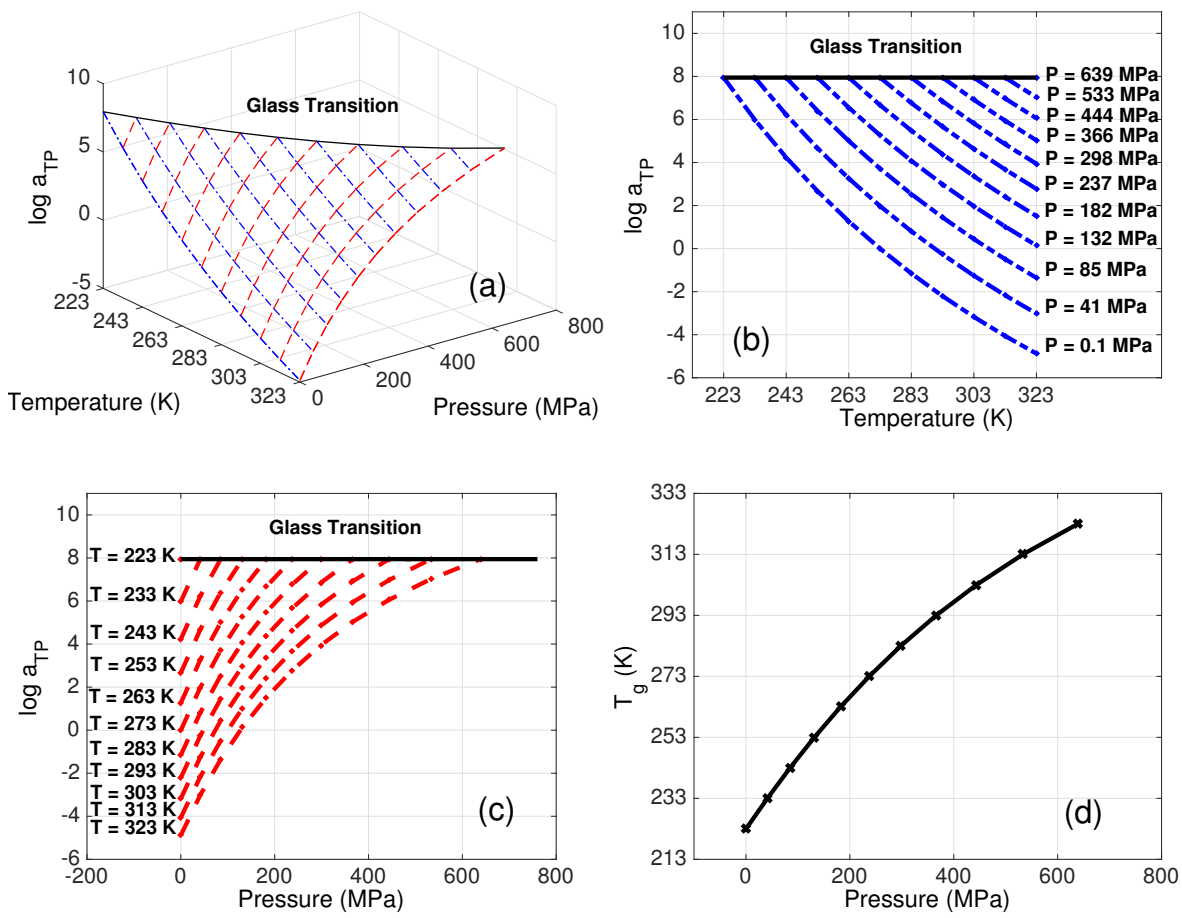

Figure 9: (a) Shift factor surface of polyurea at reference temperature and pressure $(273 \mathrm{~K}$ and $0.1 \mathrm{MPa}$ respectively). (b) Isobaric shift factor versus temperature. (c) Isothermal shift factor versus pressure. (d) Glass transition temperature, $T_{g}$ versus pressure.

\subsection{Simplification}

Even though the pressure-dependent term (equation 25) can reflect the relation between the effects of temperature and pressure on the viscoelastic behavior of polyurea and fit excellently with the experimental data, a simpler from may be more convenient for calculation and finite element models. Thus, we have tried to simplify equation 25 based on the observed trends that $c_{3}(T)$ and $c_{5}(T)$ are very close functions. The difference in the definition of these two functions, shown in equations 14 and 16, is between $l(T)$ and $l_{\phi}(T)$, i.e. the slopes of the longitudinal moduli of the polymer and that of the occupied volume (excluding free volume). Since the polymer chains occupy most of the space in a polymer, it is reasonable to assume that $l(T)$ and $l_{\phi}(T)$ are close. Note that this does not reflect the actual values of the moduli, but their dependence on pressure. Thus one may enforce that $c_{3}(T)$ and $c_{5}(T)$ are identical with small potential loss of accuracy. Furthermore, 


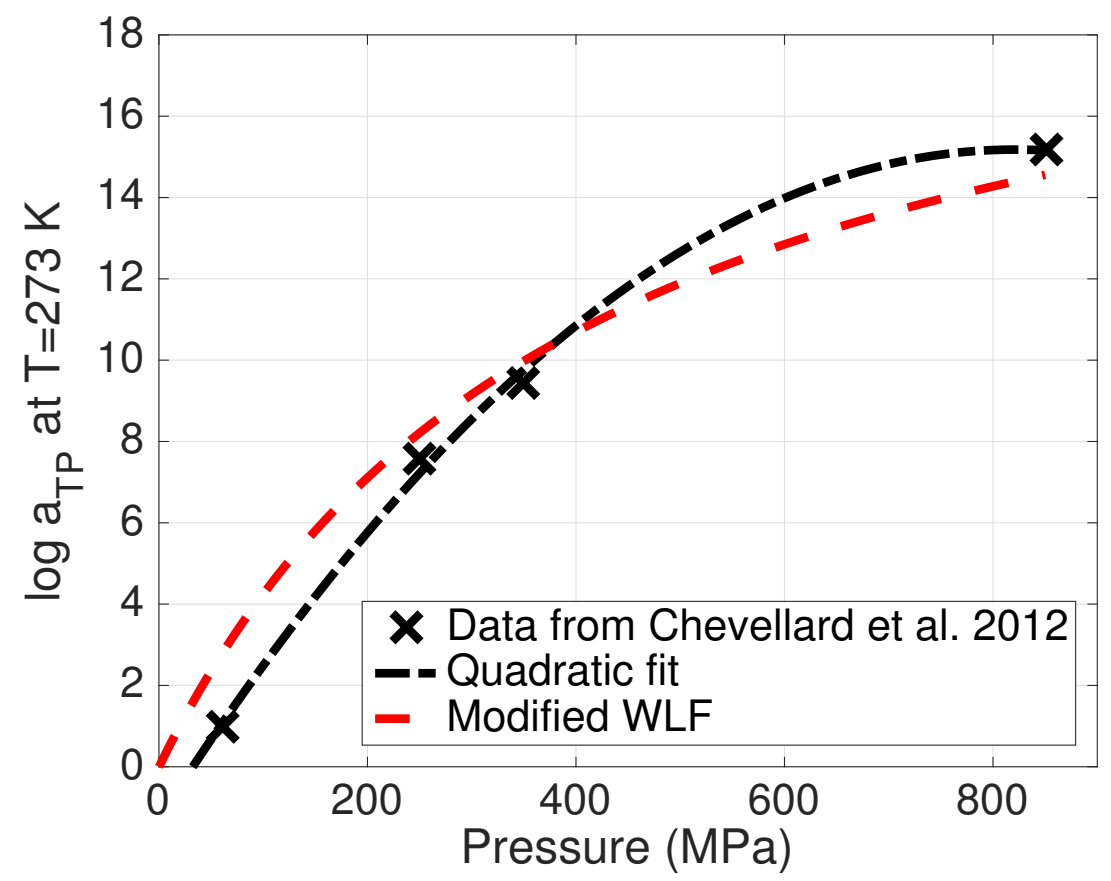

Figure 10: Comparison between the prediction from modified WLF model and experimental data obtained from the previous study on nonlinear viscoelastic behavior of polyurea by Chevellard et al [19] for reference temperature of $273 \mathrm{~K}$ and pressures at 62, 250, 370, and $850 \mathrm{MPa}$.

since $P_{o}, c_{4}(T)$, and $c_{6}(T)$ all have small values, the denominators in the logarithmic terms in equation 25 may also be neglected with negligible effect on accuracy of the model. We can then write the simplified equation for equation 25 as:

$$
\Omega^{\prime}(T, P)=c_{3}^{\prime}(T) \log \left(\frac{1+c_{4}^{\prime} P}{1+c_{6}^{\prime} P}\right),
$$

where

$$
c_{3}^{\prime}(T)=c_{31}^{\prime}\left(T-c_{32}^{\prime}\right),
$$

prime sign indicates parameters that relate to the simplified equation 26 . $c_{3}^{\prime}(T)$ has the same form as $c_{3}(T)$. We further prescribe $c_{32}^{\prime}, c_{4}^{\prime}$, and $c_{6}^{\prime}$ to have the same value as $c_{32}, c_{4}$, and $c_{6}$, respectively. It is noteworthy that when one normalizes the experimental data in Figure $6 \mathrm{~d}$ through dividing by $\left(T-c_{32}^{\prime}\right)$ in equation 27, all curves collapse and lie on top of each other (see Figure 8b). Although the temperature $c_{32}^{\prime}$ is below the glass transition temperature, it 
represents an asymptotic state for the rubbery regimes independent of the applied pressure. It will be interesting to extend such characterization and analysis to the glassy regime to see how this behavior transitions. However, is beyond the scope of the present work. Corresponding to this normalized data, equation 26 can be modified as:

$$
\frac{\Omega^{\prime}(T, P)}{T-c_{32}^{\prime}}=c_{31}^{\prime} \log \left(\frac{1+c_{4}^{\prime} P}{1+c_{6}^{\prime} P}\right) .
$$

Now one can perform another nonlinear regression analysis using equation 28 with the normalized data in Figure $8 \mathrm{~b}$ to find $c_{31}^{\prime}$. The initial guess for $c_{31}^{\prime}$ is $c_{31}$ shown in Table 1 . The result of this regression analysis is shown as solid line in Figure 8b. With $c_{31}^{\prime}$ and $I_{1}$ shown in Table $1, \alpha_{f}^{\prime}\left(P_{o}\right)$ can be calculated using equation 22 and is listed in Table 2. With $\alpha_{f}^{\prime}\left(P_{o}\right), c_{1}$, and $c_{2}$, the constants $B^{\prime}, f^{\prime}\left(T_{o}, P_{o}\right)$ can be calculated, using equations 11 , and 12 . All the primed parameters are shown in Table 2 and the rest, which are the same as the unprimed parameters are shown in Table 1. Equations 10, and 26, with parameters $c_{1}$ and $c_{2}$ in Table 1 and all the primed parameters yields the simplified form of the modified WLF equation that includes temperature and pressure effects for polyurea at reference temperature $(273 \mathrm{~K})$ and ambient pressure.

Table 2: Constants for the simplified pressure-dependence formulation $\Omega^{\prime}(T, P)$, equation 26 (The others are the same as the unprimed series used in the full form equation 25 , Table 1)

\begin{tabular}{|c|c|c|c|}
\hline \multicolumn{4}{|c|}{ Polyurea } \\
\hline \hline$B^{\prime}$ & 0.860 & $\alpha_{f}^{\prime}\left(P_{o}\right)\left(\mathrm{K}^{-1}\right)$ & $7.079 \mathrm{E}-05$ \\
\hline$f^{\prime}\left(T_{o}, P_{o}\right)$ & 0.0148 & $c_{31}^{\prime}$ & 17.99 \\
\hline
\end{tabular}

\section{Conclusion}

The original WLF equation is modified to include the effect of pressure. The increase in pressure from ambient condition is related to a temperature reduction and therefore an additional pressure-dependent term is introduced into the original WLF equation. A final closed form expression is obtained based on a thermodynamics approach. This modified WLF equation was 
calibrated for polyurea with the experimental data from the ultrasonic longitudinal wave measurements under various pressures up to $900 \mathrm{MPa}$ and temperatures as low as $243 \mathrm{~K}$. The model can be represented by a threedimensional shift surface in terms of temperature and pressure. The modified WLF equation results in a unique set of values for the parameters $\alpha_{f}\left(P_{o}\right), B$, and $f\left(T_{o}, P_{o}\right)$. A simplified closed form expression for the pressure-dependent term is also presented. It has fewer fitting parameters since all the measurements at different temperatures collapsed onto a single line once divided by $\left(T-c_{32}^{\prime}\right)$, where $c_{32}^{\prime}$ appears as an asymptotic "freezing" temperature below $T_{g}$ for the combined pressure and temperature effects on the elastomer in the rubbery regime. The full and simplified forms fit experimental data very closely. It is believed that this modified WLF equation could be used for other thermorheologically and piezorheologically simple materials under similar conditions and assumptions. Once all the necessary parameters are known, equation 10 can be used to predict the mechanical properties at any other pressure and temperature within the rubbery regime. The transition to glassy regime due to combination of increased pressure and decreased temperature is also derived based on the experimental results and can be described based on equation 10 .

\section{Acknowledgments}

The experimental work has been conducted at the Center of Excellence for Advanced Materials (CEAM) at the University of California, San Diego. This work was supported by ONR grants N00014-09-1-1126 to University of California, San Diego and N00014-13-1-0392 to University of Massachusetts, Lowell.

\section{References}

[1] J. D. Ferry, Viscoelastic properties of polymers, John Wiley \& Sons, 1980 .

[2] F. Schwarzl, A. Staverman, Time-temperature dependence of linear viscoelastic behavior, Journal of Applied Physics 23 (8) (1952) 838-843.

[3] B. Gross, Time-temperature superposition principle in relaxation theory, Journal of Applied Physics 40 (8) (1969) 3397-3397. 
[4] M. L. Williams, R. F. Landel, J. D. Ferry, The temperature dependence of relaxation mechanisms in amorphous polymers and other glassforming liquids, Journal of the American Chemical society 77 (14) (1955) 3701-3707.

[5] A. K. Doolittle, D. B. Doolittle, Studies in newtonian flow. v. further verification of the free-space viscosity equation, Journal of Applied Physics 28 (1957) 901-905.

[6] W. G. Knauss, I. Emri, Non-linear viscoelasticity based on free volume consideration, Computers \& Structures 13 (1) (1981) 123-128.

[7] W. G. Knauss, I. Emri, Volume change and the nonlinearly thermoviscoelastic constitution of polymers, Polymer Engineering \& Science 27 (1) (1987) 86-100.

[8] C. F. Popelar, K. Liechti, Multiaxial nonlinear viscoelastic characterization and modeling of a structural adhesive, Journal of Engineering Materials and Technology 119 (3) (1997) 205-210.

[9] C. Popelar, K. Liechti, A distortion-modified free volume theory for nonlinear viscoelastic behavior, Mechanics of Time-Dependent Materials 7 (2) (2003) 89-141.

[10] G. U. Losi, W. G. Knauss, Free volume theory and nonlinear thermoviscoelasticity, Polymer Engineering \& Science 32 (8) (1992) 542-557.

[11] A. Bestul, S. Chang, Excess entropy at glass transformation, The Journal of Chemical Physics 40 (12) (1964) 3731-3733.

[12] G. Adam, J. H. Gibbs, On the temperature dependence of cooperative relaxation properties in glass-forming liquids, The journal of chemical physics 43 (1) (1965) 139-146.

[13] M. Goldstein, Some thermodynamic aspects of the glass transition: Free volume, entropy, and enthalpy theories, The Journal of Chemical Physics 39 (12) (1963) 3369-3374.

[14] R. Li, Time-temperature superposition method for glass transition temperature of plastic materials, Materials Science and Engineering: A 278 (1) (2000) 36-45. 
[15] J. D. Ferry, R. A. Stratton, The free volume interpretation of the dependence of viscosities and viscoelastic relaxation times on concentration, pressure, and tensile strain, Kolloid-Zeitschrift 171 (2) (1960) 107-111.

[16] J. M. O'reilly, The effect of pressure on glass temperature and dielectric relaxation time of polyvinyl acetate, Journal of Polymer science 57 (165) (1962) 429-444.

[17] R. Fillers, N. Tschoegl, The effect of pressure on the mechanical properties of polymers, Transactions of The Society of Rheology (1957-1977) 21 (1) (1977) 51-100.

[18] N. W. Tschoegl, W. G. Knauss, I. Emri, The effect of temperature and pressure on the mechanical properties of thermo-and/or piezorheologically simple polymeric materials in thermodynamic equilibrium-a critical review, Mechanics of Time-Dependent Materials 6 (1) (2002) 53-99.

[19] G. Chevellard, K. Ravi-Chandar, K. Liechti, Modeling the nonlinear viscoelastic behavior of polyurea using a distortion modified free volume approach, Mechanics of Time-Dependent Materials 16 (2) (2012) 181203.

[20] The dow chemical company, isonate 1431 modified mdi (2001).

[21] Air products chemicals inc., polyurethane specialty products (2003).

[22] K. Holzworth, Z. Jia, A. Amirkhizi, J. Qiao, S. Nemat-Nasser, Effect of isocyanate content on thermal and mechanical properties of polyurea, Polymer 54 (12) (2013) 3079-3085.

[23] W. Nantasetphong, A. V. Amirkhizi, Z. Jia, S. Nemat-Nasser, Polyureabased composites: Ultrasonic testing and dynamic mechanical properties modeling, in: Composite Materials and Joining Technologies for Composites, Volume 7, Springer, 2013, pp. 235-243.

[24] M. Paterson, Effect of pressure on young's modulus and the glass transition in rubbers, Journal of Applied Physics 35 (1) (1964) 176-179.

[25] J. Qiao, A. V. Amirkhizi, K. Schaaf, S. Nemat-Nasser, Dynamic mechanical analysis of fly ash filled polyurea elastomer, Journal of Engineering Materials and Technology 133 (1) (2011) 011016. 
[26] W. Nantasetphong, Z. Jia, A. V. Amirkhizi, S. Nemat-Nasser, Dynamic properties of polyurea-milled glass composites part i: Experimental characterization, Mechanics of Materials 98 (2016) 142-153. 